\title{
Design \& Performance Characterization of an Anti-Backlash Spherical Joint for Micro-Manipulators
}

\author{
Taufiq Rahman ${ }^{1}$ and Nicholas Krouglicof ${ }^{2}$ \\ ${ }^{1}$ National Research Council Canada, London, ON, Canada \\ ${ }^{2}$ Faculty of Sustainable Design Engineering, University of Prince Edward Island, Charlottetown, PE, Canada
}

\begin{abstract}
Because of mating tolerances and limited range of motion, traditional implementations of spherical joints such as rod-end bearings are considered deficient in optomechatronic applications where only rotational degrees of freedom of the payload is desired. Correspondingly, when it is imperative to minimize parasitic motions in orientation manipulators composed of spherical joints, alternative designs must be explored. This paper discloses the design of an anti-backlash spherical joint with a large motion range that was specifically developed to implement a small-scale prototype of a 3PSS/S micro-manipulator. This design utilizes magnetic preloading to minimize parasitic translational motion while providing sufficient range of motion to obtain a large reachable workspace for the 3PSS/S micro-manipulator prototype. Experiments performed on the proposed design under static and dynamic load conditions showed that the goal of obtaining anti-backlash characteristics was realized.
\end{abstract}

\section{INTRODUCTION}

Micro-manipulators are employed in optomechatronics applications where motion compensation for image stabilization or high speed pointing maneuvers of optical devices such as mirrors, laser projectors, or cameras are required [1] [3]. Desired characteristics of micro-manipulators for such applications include high actuation capacity, large motion range, fast response, and precise movement in a compact form factor. Favorable mechanical properties of parallel kinematic mechanisms (PKM) can potentially deliver these requirements because the payloads are supported by multiple kinematic chains, which is more conducive to achieving precise and fast motions [4, p. 301]. Prior art in optomechatronics that involves parallel micro-manipulators with strictly orientation degrees of freedom include [5]-[7]. Although the underlying kinematic architecture of [6] is composed of revolute joints only, the prototypes in [5], [7] implemented spherical joints to realize their respective kinematic structures in two different ways. While the spherical joints in [5] were realized by traditional rod-end bearings, the design requirements of a large reachable workspace with minimal parasitic motion [8] motivated an alternative design in [7].

The authors were previously affiliated with the Memorial University of Newfoundland, NL, Canada where the presented work was completed. Currently Nicholas Krouglicof is with the Faculty of Sustainable Design Engineering, University of Prince Edward Island, Charlottetown, PE, Canada, and Taufiq Rahman is with the National Research Council Canada and is based in the Manufacturing and Automotive Innovation Hub, London, ON, Canada. All correspondences should be forwarded to: Taufiq.Rahman@nrccnrc.gc.ca
A spherical joint is typically comprised of two major components, namely a ball and a socket. In a conventional spherical joint, the ball is embedded in a spherical or conical cavity of the socket to allow only relative angular motion with three degrees of freedom. For the sake of low friction movement and easy assembly, a small gap is necessary between the mating surfaces of these two parts. However, this gap introduces backlash in the form of parasitic translational degrees of freedom, which ultimately results in inaccurate spherical motion (i.e., the constrained bodies exhibit small relative translations in addition to purely relative angular motions). In order to eliminate the backlash, alternative designs have been proposed in the literature; examples include spherical rolling joints [9] and dynamic preload adjustment ball joint [10]. Although a spherical rolling joint can provide relative motion that is virtually free of backlash, its range is generally limited. The preload adjustment ball joint requires a dedicated preload actuator and a corresponding controller. Therefore, its construction is not suitable for small-scale applications such as in micromanipulators. Furthermore, commercially available spherical joints (e.g., rod-end bearings) can offer neither backlashfree operation nor a large range of motion. This paper addresses these issues by proposing an improved design that offers a large range of motion with practically zerobacklash movement. Correspondingly, the remainder of the paper is organized as follows: the proposed design is disclosed in Section II, a corresponding prototype was developed in Section III, performance evaluation of the prototype was performed in Section IV, and finally the concluding remarks are offered in Section V.

\section{Proposed Design}

An implementation of this design is illustrated in Fig. 1. It should be mentioned that a similar design can be found in a commercial 3D printer [11]. Similar to the conventional construction of spherical joints, the proposed design includes a ball and a socket [see Fig. 1(c)]. The cylindrical socket features a continuous axial cavity between its two planar surfaces [see Fig. 1(a)]. This cavity has four distinct geometric profiles, each serving a certain function [see Fig. 1(b)]. At one end, there are two concentric spherical profiles. The outer spherical cavity features a slightly larger diameter than its adjacent cavity. Within a very tight geometric tolerance, 


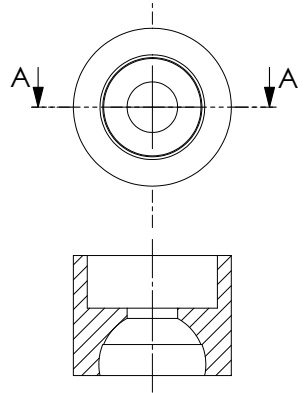

SECTION A-A

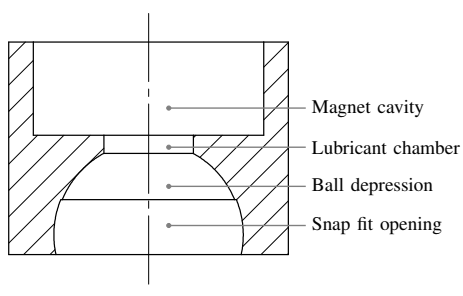

(b) Functions of the different profiles in the socket cavity.

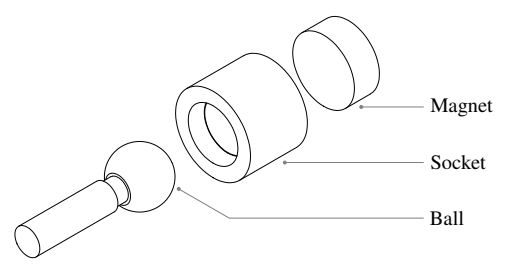

(c) Exploded view of the proposed spherical joint.

Fig. 1. Design of the magnetically loaded anti-backlash spherical joint.

the diameters of the ball and the inner spherical cavity are identical. It is also relevant to mention that the diameter of the circular opening corresponding to the outer spherical cavity is slightly smaller than the diameter of the ball, which creates a snap fit between the socket and the ball. The snap fit design increases the capacity of the joint in terms of the maximum load it can support; i.e., the force needed to completely separate the ball and the socket assembly. The corresponding breakaway force was experimentally confirmed to be sufficient for the dynamic loads that may occur in a small-scale orientation manipulator. Of the two cylindrical cavities at the other end of the socket, the outer cavity accommodates an axially magnetized cylindrical magnet and the inner cavity serves as a lubricant chamber. The attractive force between the magnet and the ferromagnetic ball acts as an anti-backlash preloading. Thus, backlash-free motion is obtained as long as the load on the joint does not exceed the magnetic preloading. Although a stronger magnet may seem desirable to extend the joint's capacity, the greater normal force from a large magnetic preload results in increased friction. Therefore, the strength of the magnet should be chosen as a compromise between the achievable range of backlash-free motion and the maximum allowable friction in the joint.

\section{Prototype Development}

Delrin is a thermoplastic polymer that offers excellent abrasion resistance, low coefficient of friction, and high heat resistance. Because of these favorable mechanical properties, the socket of the proposed spherical joint was manufactured from this material. A precision $\mathrm{CNC}$ machining process was employed to ensure the geometrical accuracy of the spherical cavities in the prototyped socket. The inner spherical profile of the socket cavity was dimensioned to mate with a

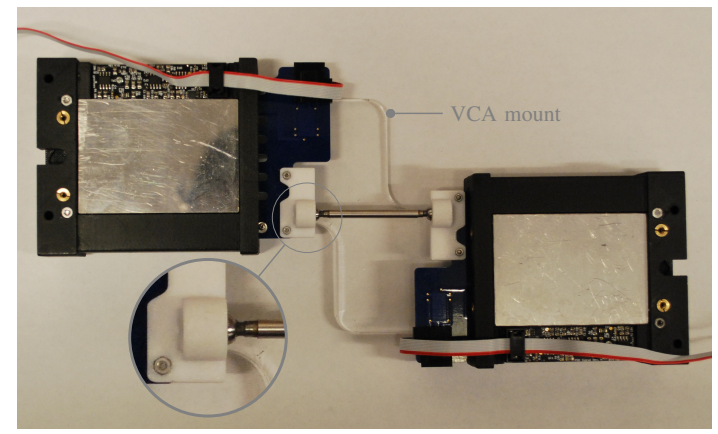

(a) Experimental setup for quantifying anti-backlash characteristics under static conditions. Inset: proposed spherical joint prototype.

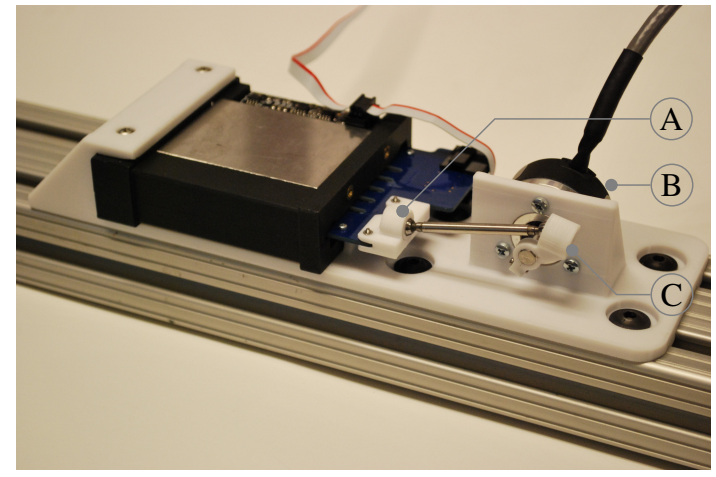

(b) Experimental setup for determining motion transmission characteristics under dynamic conditions. A: slider, B: encoder, C: crank.

Fig. 2. Experimental evaluation of the proposed spherical joint.

commercially available tooling ball [see Fig. 1(c)] made of magnetic stainless steel. Since a tooling ball is typically used to establish a reference point by measuring hole centerlines in precision machining tasks, it is manufactured to a tight tolerance. Specifically, the tooling ball used in this prototype features a diameter of $\frac{1}{4}$ inch with a tolerance $\pm 0.2 \mathrm{mil}$. It is obvious that the construction of the proposed design offers the full range of the torsional motion. In addition, it can provide a maximum tilt angle of $54^{\circ}$ while covering the entire range of the azimuth angle. See [12] for representing the space of rotation in terms of tilt and torsion angles.

\section{EXPERIMENTAL EVALUATION}

In order to quantify the performance of the proposed design, two separate experiments were performed. The purpose of the first experiment was to demonstrate the anti-backlash properties under static loading. In contrast, the second experiment was conducted to quantify the dynamic motion transmission characteristics.

\section{A. Static Performance Characterization}

The experimental setup corresponding to the first experiment is shown in Fig. 2(a), which was comprised of two voice coil actuators (VCA) from [7]. These two VCAs were mounted on a rigid platform so that their axes were aligned. The integrated position sensor with sub-micron resolution was proved to be immensely useful for identifying the backlash 
characteristics of the proposed design. In this experiment, one actuator was arbitrarily chosen to be the driver VCA. The other VCA (i.e., follower) remained constrained to the driver by a shaft. At each end of the shaft, a spherical joint provided the connecting interface between the actuator piston and the shaft body. The ball and the socket of the the spherical joint adjacent to the driver VCA were rigidly bonded together by using epoxy. Consequently, the other joint alone compensated for any misalignment between the axes of the actuators. Furthermore, restricting the motion of one spherical joint ensured that any backlash in the system was contributed by a single joint. Although the coil of the follower VCA did not receive any power, its position sensor remained active to provide its location. In addition, the position of the driver VCA was controlled in order to characterize the performance of the proposed spherical joint.

In each of the experimental trials, the driver VCA traveled a distance of $1.84 \mathrm{~mm}(\approx 10000$ counts from the ADC embedded in the position sensor) from either direction (i.e., inward or outward motion) to reach and maintain a predefined target position. The mid-stroke of the driver VCA was arbitrarily chosen as the target position. Sufficient time was allowed after each experimental movement so that the driver VCA could completely come to a rest. After the driver VCA motion was settled, the positions of the two actuators were sampled for 1000 times. Since the position controller of the driver VCA failed to reach the target position with a minimum steady state error in some trials, they were correspondingly discarded from the post-experiment analysis. In specific terms, the acceptance criterion in this regard dictated that the standard deviation of the 1000 position samples must be less than $0.18 \mu \mathrm{m}(\approx 1.0$ ADC counts $)$, and the corresponding mean must be within $0.37 \mu \mathrm{m}(\approx 2.0$ ADC counts $)$ of the target position. A total of 100 such successful trials were recorded. The first fifty trials were conducted by performing inward motion, and outward motion was employed to execute the remaining fifty trials. These trials constituted the treatment group of the experiment. In addition, a control group was established by recording 100 more successful trials with no preload magnet in the spherical joint being examined.

The recorded experimental data is graphically presented in Fig. 3. The zero positions of the two actuators in each experiment group were provided by respective group means. The presented data exhibits four distinct clusters. It can be observed that the data points in each individual cluster were obtained by approaching the target position from the same direction (i.e., outward or inward motion). Furthermore, a comparison between the distributions of the data points from the two experiment groups indicates that the magnetic preloading in the proposed design indeed minimizes backlash. In specific terms, the mean positions of the follower VCA from the treatment group and the control group are respectively $\pm 2.3750 \mu \mathrm{m}$ and $\pm 4.5759 \mu \mathrm{m}$. Since this experiment did not account for other sources of error such as measurement error or the compliance of the experimental setup, it is unclear whether the small magnitude of the backlash observed in the treatment group was entirely caused by the spherical joint

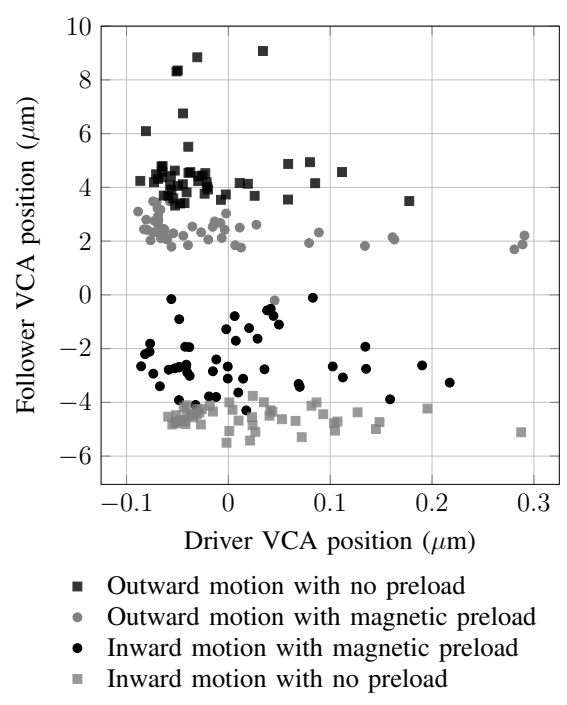

Fig. 3. Experimental observations in the static repeatability test.

itself. Nonetheless, the proposed design was shown to provide nearly backlash-free motion under static conditions.

\section{B. Dynamic Motion Transmission Characteristics}

The proposed spherical joint was subjected to a negligible static load in the previous experiment. In order to determine the performance of the proposed design under dynamic loading, a second experiment was conducted employing the experimental setup shown in Fig. 2(b). This experimental setup can be characterized as a classic slider-crank mechanism, where the two revolute joints were replaced by two proposed spherical joints. In addition, the proposed VCA from [7] served as the slider and the motion generator.

An encoder was used to determine the crank angle and the integrated position sensor provided the slider position. For the sake of this experiment, it was necessary to obtain a mathematical model that provides the slider position as a function of the crank angle. To this end, the corresponding analytical model defined by the geometric parameters (e.g., crank radius, connecting rod length, offset, etc.) of this slider-crank mechanism can be employed. However, the cited geometric parameters must be estimated by metrological means with acceptable accuracy. Alternatively, a simpler solution can be formulated by employing an empirical model derived from a finite set of experimental observations. Specifically, a ninth order polynomial model estimated by least squares curve fitting was commissioned to provide an accurate representation of motion of the experimental setup.

It should be mentioned that the position sensor of the VCA is a first order filter with a time constant of $100 \mu \mathrm{s}$ [13]. Due to its dynamics, the signal provided by the sensor lags the true position by a small amount, especially when the VCA is in motion. In order to demonstrate the effects of sensor dynamics, the slider was powered by a step input with a magnitude of 24.0 Volts. The corresponding slider positions and the encoder positions were recorded. From these recorded data, two time series of crank angles are presented in Fig. 4. 


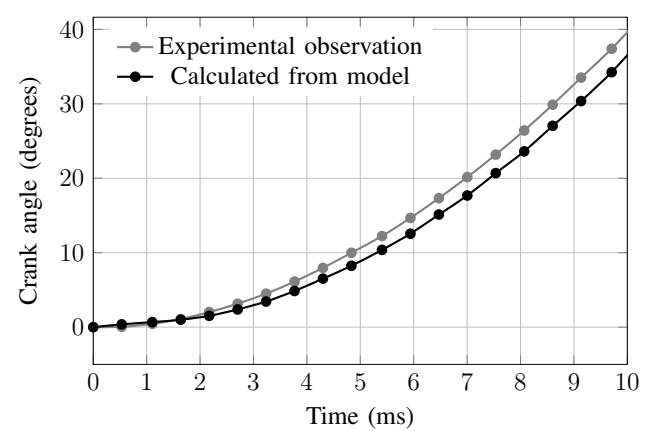

Fig. 4. Experimental demonstration of position sensor dynamics.

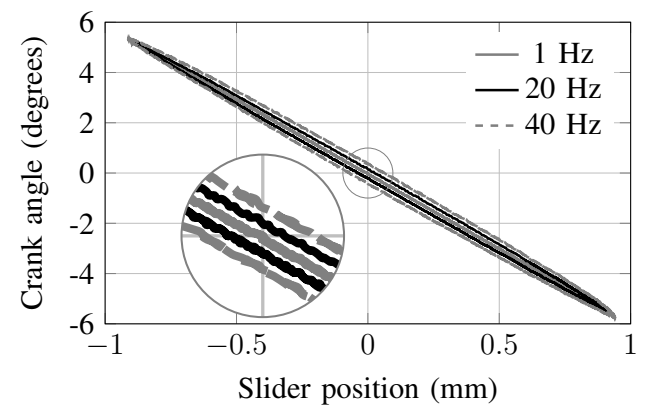

Fig. 5. Experimental results obtained from the slider-crank mechanism.

The experimental observation in Fig. 4 refers to the crank angles obtained from the encoder. In addition, the second time series was calculated from the recorded slider positions employing the aforementioned empirical model. It is relevant to mention that the encoder can be regarded as a pure gain without any lag between its input and output. The time lag between the two time series of crank angles demonstrates the dynamics of the position sensor. The entire stroke of the VCA was employed for this exercise.

In order to impose dynamic loading on the two spherical joints in the experimental setup, the slider (i.e., VCA) followed a sinusoidal position signal oscillating about its midstroke. The corresponding position profile is characterized by an amplitude of $920.45 \mu \mathrm{m}(\approx 5000$ ADC counts $)$. The system responses for three different frequencies were recorded and are graphically presented in Fig. 5. When the input frequency was low (i.e., $1.0 \mathrm{~Hz}$ ), the inertial forces on the two spherical joints were negligible. Furthermore, the dynamics of the position sensor did not significantly effect the experimental observations because the corresponding slider velocity was relatively low. However, at higher frequencies, the crank angles showed obvious hysteresis-like behavior. While it was partly caused by the dynamics of the position sensor, the inertial forces associated with the oscillating motion at high frequencies also contributed by overcoming the magnetic preloads in the two spherical joints. Therefore, it can be concluded that the anti-backlash characteristics of the proposed design is not infinite. However, since the proposed design was primarily developed for constructing small-scale PKMs, its performance, as quantified by experimental means, is considered to be sufficient for the desired application.

\section{CONClusion}

This paper describes an alternative design for spherical joints which was specifically developed for micromanipulators in optomechatronic applications. Furthermore, the proposed design was prototyped, and was experimentally evaluated under static and dynamic loading conditions. Experimental results clearly indicate that magnetic preloading in the proposed design effectuates anti-backlash characteristics. The compact design of the spherical joint was found particularly suitable for integration into micro-manipulator prototypes.

\section{REFERENCES}

[1] C. H. Tee, J. H. Lee, S. H. Kim, and Y. K. Kwak, "Development of beam rotating actuator based on voice coil motor type for multibeam optical disc system," in Intelligent Robots and Systems, 1999. IROS'99. Proceedings. 1999 IEEE/RSJ International Conference on, vol. 3. IEEE, 1999, pp. 1884-1889.

[2] P. Gandhi and S. Deshmukh, "A 2d optomechanical focused laser spot scanner: analysis and experimental results for microstereolithography," Journal of Micromechanics and Microengineering, vol. 20, no. 1, p. 015035, 2010.

[3] M.-G. Song, Y.-J. Hur, N.-C. Park, K.-S. Park, Y.-P. Park, S.-C. Lim, and J.-H. Park, "Design of a voice-coil actuator for optical image stabilization based on genetic algorithm," Magnetics, IEEE Transactions on, vol. 45, no. 10, pp. 4558-4561, 2009.

[4] J. Merlet, Parallel Robots, 2nd ed., ser. Solid mechanics and its applications. Dordrecht, The Netherlands: Springer, 2006.

[5] T. Villgrattner and H. Ulbrich, "Design and control of a compact high-dynamic camera-orientation system," Mechatronics, IEEE/ASME Transactions on, vol. 16, no. 2, pp. 221-231, 2011.

[6] C. M. Gosselin and É. St-Pierre, "Development and experimentation of a fast 3-dof camera-orienting device," The International Journal of Robotics Research, vol. 16, no. 5, pp. 619-630, 1997.

[7] T. Rahman, "Design synthesis \& prototype implementation of parallel orientation manipulators for optomechatronic applications," Ph.D. dissertation, Memorial University of Newfoundland, 2016.

[8] J. Carretero, R. Podhorodeski, M. Nahon, and C. M. Gosselin, "Kinematic analysis and optimization of a new three degree-of-freedom spatial parallel manipulator," Journal of Mechanical Design, vol. 122, no. 1, pp. 17-24, 2000.

[9] S. Noguchi, H. Aramaki, and T. Kanada, "The development of pivot bearing with multiple degrees of freedom: 1st report: Prototype of pivot bearing with two degrees of freedom," Precision Engineering, vol. 30, no. 4, pp. 373 - 380, 2006. [Online]. Available: http://www.sciencedirect.com/science/article/pii/S0141635906000067

[10] R. A. Kendall, "Ball joint with dynamic preload adjustment," Oct. 1 1991, uS Patent 5,052,844.

[11] "deltaRocket - fast and accurate 3D printing," retrieved on March 20, 2015. [Online]. Available: http://deltarocket.com/

[12] I. A. Bonev and C. M. Gosselin, "Analytical determination of the workspace of symmetrical spherical parallel mechanisms," Robotics, IEEE Transactions on, vol. 22, no. 5, pp. 1011-1017, 2006.

[13] N. Krouglicof, M. Morgan, N. Pansare, T. Rahman, and D. Hicks, "Development of a novel pcb-based voice coil actuator for optomechatronic applications," in Intelligent Robots and Systems (IROS), 2013 IEEE/RSJ International Conference on, 2013. 